\title{
Analysis of the recent Plio-Quaternary deformation and the correlation with the focal mechanisms of the significant earthquakes in the Bokoya, Ras Tarf (Eastern Rif)
}

\author{
A. El Mahsani ${ }^{1}$ and L. Ait Brahim ${ }^{1}$ \\ ${ }^{1}$ Université Mohammed V, Faculté des sciences Département sciences de la terre, Unité de recherche GEORISK et \\ LGRN, 4 Avenue Ibn Battouta Rabat-Agdal, BP 1014, Rabat, Maroc.
}

\begin{abstract}
Relative movements of microplates developed between the African and Eurasian plate characterize the continuity of the tectonic movements in Westerner of the Mediterranean Sea. The convergence movements between these plates, at the origin of the current structuring in oriental Rif. The recent tectonics in the south ripple of Alboran Sea, translated by an important seismic activity, In the North of Morocco, exactly in the Al Hoceima region, considered as zone of plates border with important seismicity. The structural analysis made in this paper and the interpretation of focals mechanisms earthquakes characteristics in the Al Hoceima region, has allows to deduct the main directions of faults, which we compared with the results of the satellite images. This study we has provides to demonstrate the directions of faults which are able to generate earthquakes in the future. The direction NE-SW represents the overall faults dominant in oriental Rif, this major direction coincides with the breaks of surface. A reduced number of faults shows a generall direction NNE-SSO to N-S through the Quaternary formations in the $\mathrm{NO}$ of $\mathrm{Al}$ Hoceima city, the direction $\mathrm{NO}$ does not show a good correlation with the directions of surface rupture, as well as with the deducted directions from focals mechanisms. This study drives us to propose that faults of direction NNE-SSO to NE-SO, represent the major directions of the faults in the Al Hoceima region, which represents the main cause of the seismic activity in the region. Key words: Al Hoceima, Quaternary, Active faults, Focal mechanisms.
\end{abstract}

\section{Introduction}

La chaine alpine du Rif est issue du rapprochement entre les plaques Africaines et Eurasiatique qui subissent un rapprochement oblique dextre avec un taux de déplacement de $4.5 \mathrm{~mm} /$ année [1]. Dans le cadre de ce rapprochement la région d'Al Hoceima a connu de nombreux séismes violents dans le passé [2]. Récemment des séismes destructeurs ont eu lieu : 26 mai1994, 24 février 2004 et celui du 25 janvier 2016. Par conséquent, la région d'Al Hoceima est considérée comme la zone la plus active sismiquement au Maroc.

Les différents travaux effectués et méthodes utilisées (Mécanismes au foyer, Radar insar etc) pour déterminer les directions des failles responsables des séismes destructeurs dans la région d'Al Hoceima de 1994, 2004 et 2016 proposent des solutions différentes entre elles et surtout avec les déformations cosismiques de surface développées lors de ses séismes [3-7]. Dans le but de lever cette ambiguité, nous avons d'une part repris l'analyse de nos relevés de terrain cartographiés lors des séismes de 1994 et 2004 et d'autre part effectué de récentes missions de terrain dans les formations géologiques récentes plio-quaternaire des régions limitrophes de la région d'Al Hoceima.

\section{Présentation de la zone d'étude}

La zone d'étude se situe au nord du Maroc, elle correspond à l'extrémité orientale de la chaine du Rif. Elle est limitée au nord par la partie méridionale de la mer d'Alboran. Les formations géologiques dans la région se présentent sous forme d'empilement de nappes de charriages à contacts anormaux E-W à vergence Sud [8]. Les principaux domaines structuraux présents dans la région d'étude sont : le massif des Bokoya marqué par un empilement de plusieurs unités structurales séparées les unes des autres par des contacts anormaux E-W [8]. Il est dominé par les formations carbonatées de la dorsale externe, sur lesquelles reposent sous forme de klippes tectoniques des terrains appartenant à la dorsale calcaire 
interne ainsi que les nappes Paléozoïques Ghomarides [8]. L'ensemble du massif chevauche vers le sud la série des flyschs gréseux de Tizirène par le biais d'une couche marneuse du Prédorsalien (semelle tertiaire), elle-même charriée sur les unités du Rif externe au sud [9]. Les formations post nappes correspondent aux bassins post nappes de Boudinar (tortono-messinien) avec le volcanisme de Ras Tarf qui est un massif andésitique d'âge miocène (première manifestation volcanique qui affecte la marge méditerranéenne marocaine postérieurement aux phases majeures de l'orogenèse alpine lié à un modèle de subduction ) et celui du bassin d'Al Hoceima (baie de nekor) à remplissage quaternaire.

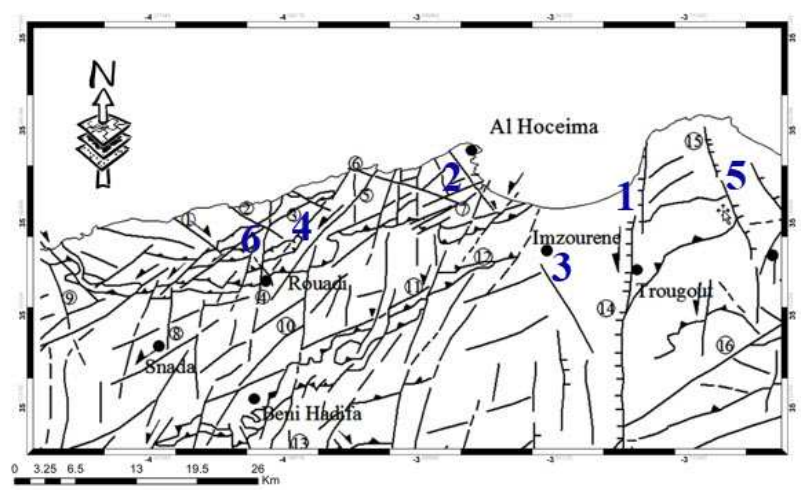

Fig. 1. Principales failles dans la région d'Al Hoceima (1: Faille Trougout, 2: Faille Boujibar, 3: Faille d'Imzouren, 4: Faille de Boussekour, 5: Faille Boudinar, 6 : Faille Rouadi).

Ces différentes unités structurales sont recoupées par des failles majeures transverses : du Nekor (NE-SW), d'Al-Hoceima (ENE-WSW), d'Imzouren (NNW-SSE), d'El Hammam (NNW-SSE), de Rouadi, (NNE-SSW), de Boussekour (NNE-SSW et celle d'ajdir WNW-ESE) [10, $4,8,11,12,13,5,14,6]$.

La région d'Al Hoceima est la zone la plus active sismiquement au Maroc avec une forte concentration d'épicentres (figure 2). Cette sismicité est la conséquence de l'activité tectonique complexe associée à la convergence Nord-Sud entre les plaques Afrique et Eurasie [3,15].

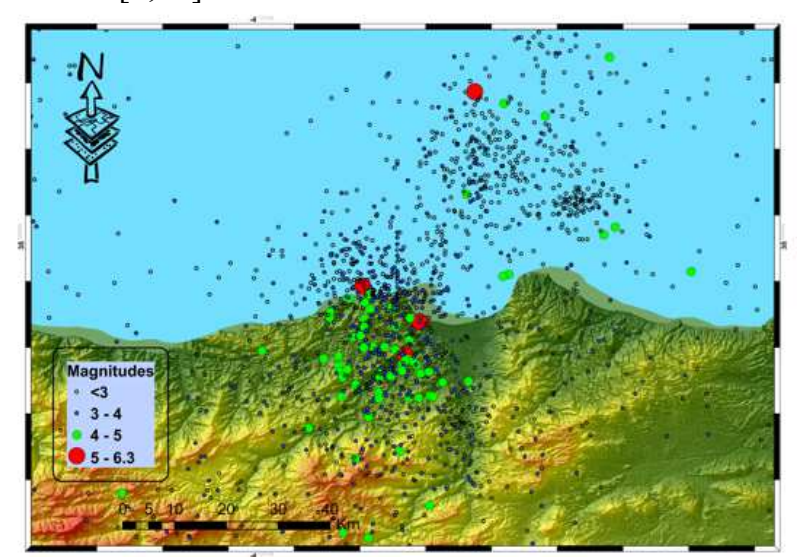

Fig. 2. Carte de la distribution des séismes, dans la région d'Al Hoceima entre 1900 et 2016.
Les principaux accidents cartographiques recensés responsables de la structuration de la région d'Al Hoceima qui recoupent les principaux fronts de chevauchement (E-W) sont (figure 1): Trougout (N-S), Imzouren (NNW-SSE), Hammam (NNW-SSE), Rouadi, (NNE-SSW), Boussekour (NNE-SSW), Nekor (NE-SW). $[4,5,6,8,11,12,14]$.

\section{Matériels et méthodes}

La méthodologie adoptée dans cette étude est basée sur le traitement des images satellitaires de haute résolution et surtout l'analyse de la déformation cassante dans les formations plio-quaternaires (séries marines et continentales) de la région d'Al Hoceima suite aux nouveaux affleurements dégagées par la route côtière (la rocade). L'analyse des images satellitaires pour l'extraction des linéaments s'est faite par une série de traitements afin d'améliorer la qualité des résultats. La prise en compte de l'analyse du réseau hydrographique en termes d'anomalies et de perturbation était d'un apport considérable dans la détermination des segments des failles actifs ayant guidés le réseau hydrographique de la région d'Al hoceima $[16,17]$.

\section{Carte des linéaments issus de l'image satellitaire}

Les résultats issus de l'analyse des images satellitaires dans la région d'Al Hoceima montre une prédominance des directions NE-SW à ENE-WSW à secondairement la direction submeridienne.

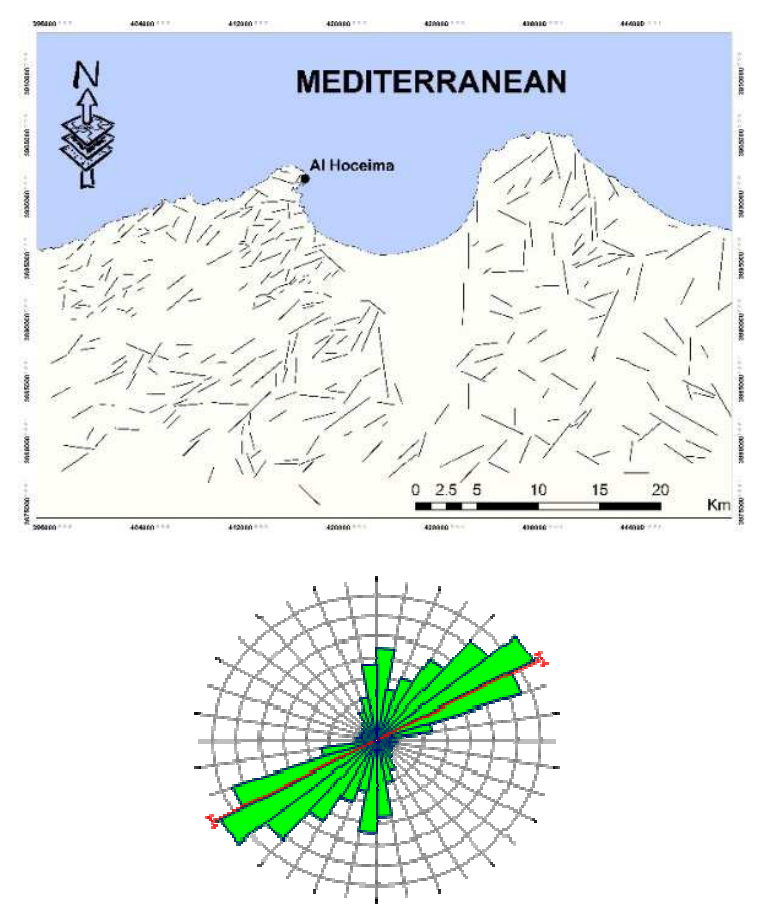

Fig. 3. Carte des linéaments issus de l'image satellitaire Landsat 8 Oli. La rosace des directions des linéaments montre les pourcentages suivants : N170-N010 25\%, N030-N050 27 $\%$, N060-N070 $33 \%$. 


\section{Analyse de la déformation récente dans la région d'Al Hoceima}

La reprise de nos données de terrain (que nous n'avons pas publiées), associées aux récentes observations effectuées dans les formations géologiques récentes plio-quaternaire grâce aux nouveaux affleurements dégagées par la route côtière (la rocade), nous ont permis d'identifier et d'analyser de nouveau la déformation cassante dans les régions limitrophes de la région d'Al Hoceima.

\subsection{Les formations quaternaires marines situées au NE de la ville d'Al-Hoceima}

Le secteur d'Al Hoceima se caractérise par la présence des failles normales et des fractures de directions NNW-SSE à NE-SW (perpendiculaire à l'orientation de la côte). La majorité des failles montre un pendage sub-vertical $\left(70^{\circ}\right.$ à $\left.90^{\circ}\right)$. Nous avons observé dans cette zone de nombreuses manifestations d'une activité tectonique récente, voire actuelle, de la faille d'Ajdir. Des fractures de direction N-S affectent les sables quaternaires et les schistes du socle paléozoïque (figure 4. B). Dans les sables, ces fractures N-S forment un réseau de failles normales néoformées à pendage opposé (figure 4. A). En surface, nous avons observé aussi, des fractures de directions subméridiennes au niveau de calcrètes récentes qui s'organisent sous forme de fentes de tension, parfois disposées en echelon compatibles avec un composante décrochante dextre. Le long de la côte nous avons identifiées des failles normales subméridiennes (N20 à N160) qui décalent les sables quaternaires de plusieurs dizaines de $\mathrm{cm}$ (figures 5 et 6 ). Parmi ces failles normales, celle de direction N160 qui décale avec un rejet vertical de l'ordre de $60 \mathrm{~cm}$ un niveau conglomératique à gros galets côtiers (figure 5).

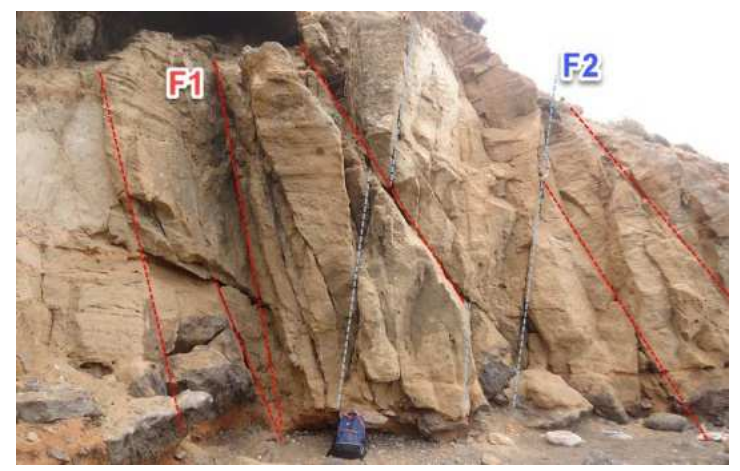

Fig. 4.A. Formation sableuse quaternaire affectée par des fractures de direction N170 à N190 à pendage opposé F1, F2.

\subsection{Les formations aux WNW d'Imzouren}

Des niveaux récents de colluvions au niveau de l'oued Rhis au NW d'Imzouren, ainsi que des terrains villafranchiens nous ont permis d'observer des indices de déformations quaternaires à actuelles, liées à l'activité des failles actives dans le Rif oriental. Parmi ces indices, les plus représentatifs, les nombreuses failles normales de direction N140 à N150 que nous avons observé à l'ouest $\mathrm{du}$ tracé cartographique de la faille d'Ajdir-Imzouren (figure 1).

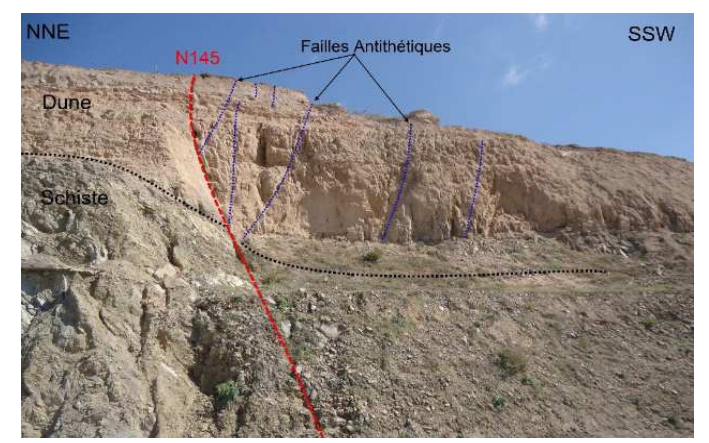

Fig. 4.B. faille normale de direction N145, affectant une dune sableuse et le socle Paléozoïque.

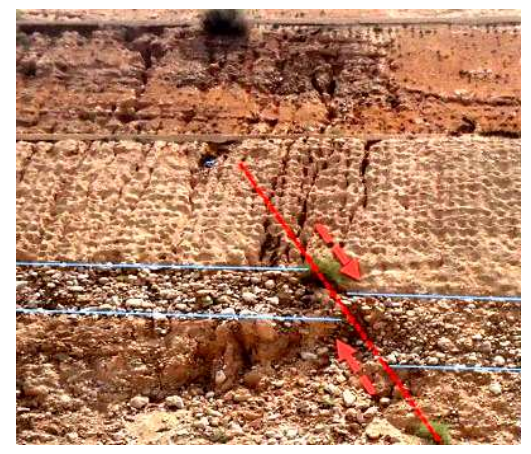

Fig. 5. Le long de la cote, une faille normale N160, 80W décale un niveau conglomératique quaternaire avec un rejet vertical de $60 \mathrm{~cm}$.

Ces failles qui présentent aussi bien des pendages vers le SW que vers le NE (figure 7), affectent les terrains mésozoïques de la dorsale calcaire, les formations flyschoïde de Tizirène, et se prolongent dans les colluvions quaternaires sans atteindre systématiquement la surface. Dans plusieurs cas, elles affectent les calcrètes à la base des sols actuels. Il est important de noter ici que ce type de structure n'a pas été rencontré à proximité de la zone de faille d'Ajdir, mais dans une large zone située plus loin à l'Est (de la faille d'Ajdir). Ceci nous amène à penser que l'activité de cette structure n'est pas aujourd'hui cantonnée à un plan de faille cartographiquement bien défini, mais à des structures secondaires parallèles qui se distribuent sur un large couloir de faille de plusieurs centaines de mètres, voire quelques kilomètres.

\subsection{Les régions de Trougout et Ras-tarf}

Le soulèvement récent de l'extrémité Nord du massif volcanique de Ras Tarf a déjà été décrit grâce à l'étagement de terrasses marines étagées [5]. Ce soulèvement est guidé par la faille NS du massif de Trougout (qui est responsable aussi de l'effondrement du bassin d'Al Hoceima). Les formations récentes au NE de la faille de Trougout, montrent les traces d'une activité tectonique récente, grâce aux récents affleurements dégagés lors de la construction de la route 
(la rocade) qui longe la côte méditerranéenne. Ainsi, nous avons observé des plans de failles, de directions NNWSSE à NNE-SSW (figure 8), qui mettent en contact des colluvions quaternaires avec les séries volcaniques tortono-messiniennes du Ras Tarf. La présence de facies mylonitique pulvérulent au niveau des plans de ses failles atteste de leur récente activité. Les miroirs de ces failles récemment dégagés montrent des stries subhorizontales et des tectoglyphes qui attestent du rejeu en décrochement senestre récent postérieurement au jeu en faille normale à fort escarpement vertical dans la topographie lors des phases tortono-messiniennes.

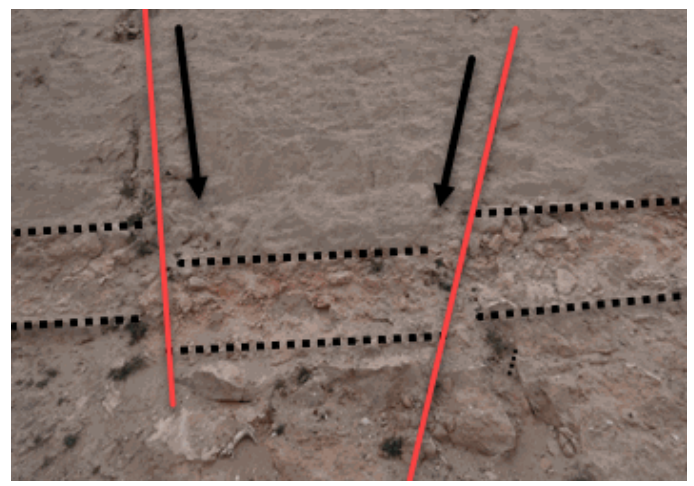

Fig. 6. Deux failles normales de direction N20 à regard opposé affectent une formation sableuse en décalant un niveau conglomératique

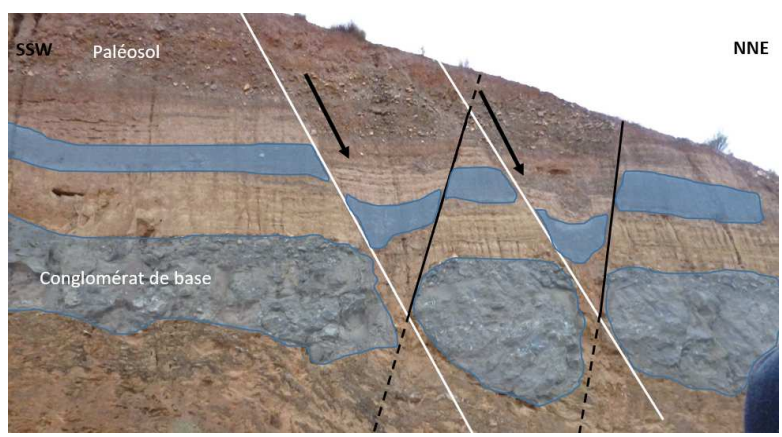

Fig. 7. Failles normales à pendage opposé de direction N100, affectant une formation du Villafranchien.
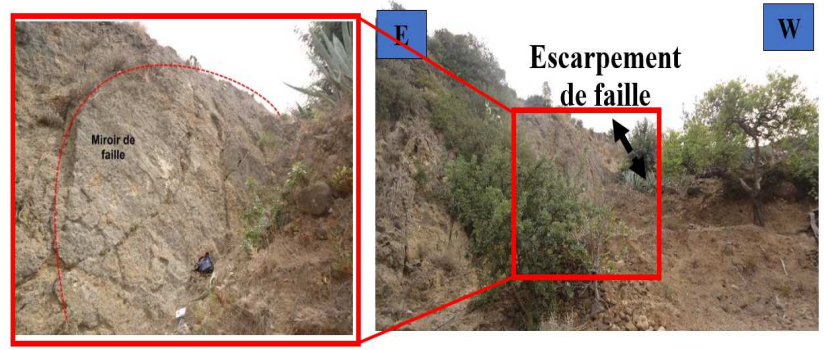

Fig. 8. Miroir de la faille de Trougout, dans la région d'Ahadad, B. l'escarpement de la terrasse de l'oued par le jeu de la faille de Trougout.

\section{Ruptures de surface développées lors des séismes de 1994 et 2004}

Les déformations de surface que nous avons cartographiées lors du séisme de 1994 se répartissent sur plus de $20 \mathrm{kms}$ le long de la faille de Boussekour (de la côte méditerranéenne jusqu'à Rouadi). Elles correspondant à des fractures de direction NE-SW à légère composante inverse avec localement des fentes de tensions en échelon qui indiquent un jeu décrochant senestre [18].

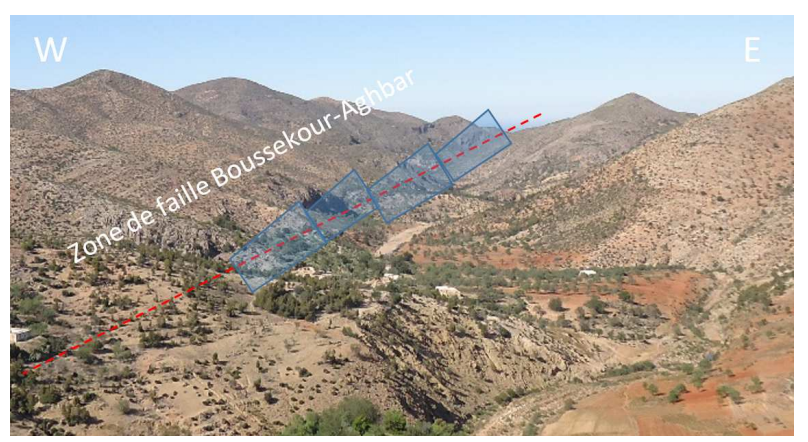

Fig. 9. Le tracé de la faille de Boussekour-Aghbar qui montre une direction (NE-SW), responsable de l'évènement sismique du 26 Mai 1994.

Pour le séisme. L'analyse des mécanismes au foyer de ce séisme, propose [19] que la meilleure solution correspond au mouvement de décrochement à composante inverse du premier plan nodal sénestre orienté N22E. [6] proposent $\mathrm{N} 20^{\circ} \mathrm{E},[20]$ proposent une rupture complexe sur des failles de direction $\mathrm{N} 175^{\circ} \mathrm{E}$ et $\mathrm{N} 150^{\circ} \mathrm{E}$. La direction déduite par les données InSAR (interférogrammes) réalisées par [21], proposent un plan de faille de direction N23E avec un pendage de $77^{\circ}$ vers le SE. Les déformations de surface que nous avons cartographiées lors du séisme de 2004 se répartissent sur plus de $60 \mathrm{kms}$ (entre Beni-Abdellah et Ajdir) (figure 11) dans la nappe de Tizirene, l'unité de kétama et les alluvions recents de l'oued Rhis (avec développement de liquéfactions locales). Elles correspondant à des fractures de direction NE-SW avec localement des fentes de tensions en échelon qui indiquent clairement un jeu decrochant senestre [4]. Les mécanismes au foyer montrent tous un glissement en décrochement sur des plans nodaux subverticaux orientés NNE-SSW et WNW-ESE (SED, HRV, IGN, IAG, USGS, EMSC) (figure 10).

Le plan nodal de faille de décrochement senestre, $\mathrm{N} 11^{\circ} \mathrm{E}, 74^{\circ}$ a été préféré par [22]. La répartition des répliques montrent deux essaims de direction NESW et NW-SE. Leurs mécanismes au foyer indiquent un jeu décrochant senetre pour les NE-SW et dextre pour les NW-SE [23]. Cette solution des deux failles a été reprise et retenue par [24] avec distance entre les deux failles estimée à $1,5 \mathrm{~km}$. La direction déduite par les données InSAR (interférogrammes) réalisées par [21], proposent un plan de faille de direction N23E avec un pendage de $77^{\circ}$ vers le SE. Devant cette complexité de la distribution des répliques et des solutions proposés par les 
mécanismes au foyer, l'analyse des données InSAR [25] n'a pas pu retenir le mouvement de décrochement N-S a N15E, comme unique plan responsable du séisme de 2004, car les interférogrammes cosismiques font ressortir dans le même pourcentage aussi la direction NW-SE.

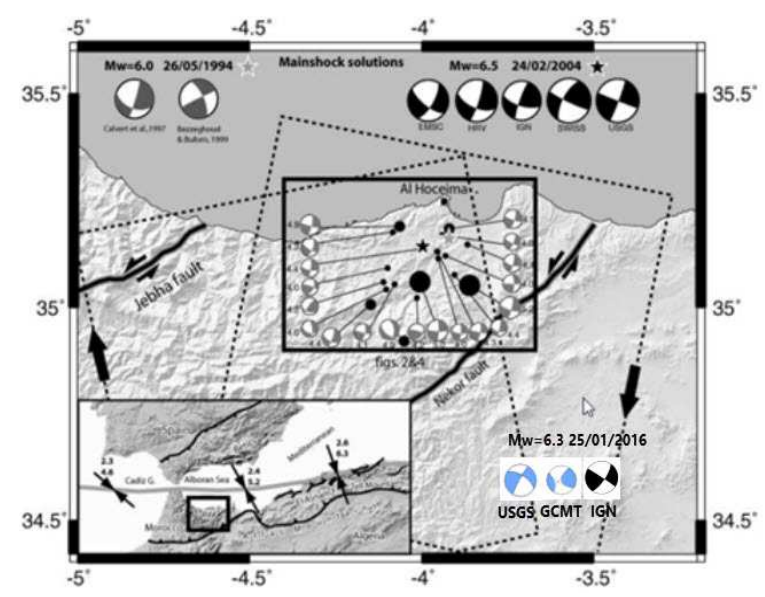

Fig. 10. Mécanismes au foyer calculés pour les évènements sismiques du 1994, 2004 et 2016 (EMSC, HRV, IGN, SWISS, USGS, GCMT). Modifié [23].

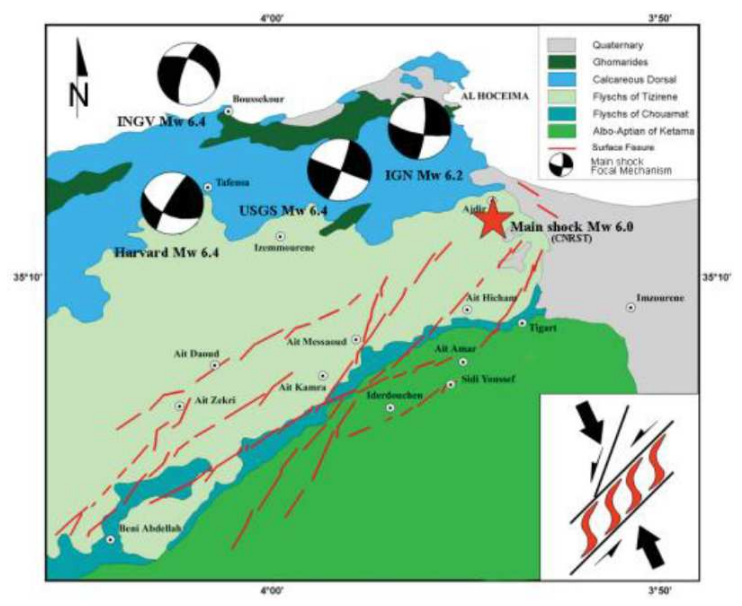

Fig. 11. Ruptures de surface de direction NE-SW cartographiées après le séisme du 2004, [17].

\section{Résultats et discussions}

Les résultats obtenus donnent ainsi trois grandes catégories de directions, NS à NNE-SSW, NE-SW et NNW-SSE à NW-SE. La direction NE-SW coöncide avec les résultats issus de l'analyse des images satellitaires et correspond à la direction la plus abondante dans la région d'Al Hoceima (figure 3). Cette direction est en accord avec la direction de la faille de Boussekour Aghbar (figure 9) responsable de l'événement sismique de 1994, ainsi que les fracturations de surface causées par le séisme du 2004 (figure 11). L'analyse des mécanismes au foyer des séismes majeurs (figure 10), a permis de mettre en évidence deux directions de failles en profondeur, NESW et NW-SE [24]. On note que les données issues de la méthode de sismique réflexion haute résolution ont permis de mettre en évidence l'existence des failles dans le large de la mer d'Al Hoceima de directions NE-SW [26]. Cela nous ramène à proposer que l'activité sismique dans la région d'Al Hoceima est guidée par des failles de direction allant de N-S a NE-SW (avec une prédominance des failles de direction NE-SW qui restent les plus abondantes dans la région d'Al Hoceima et de la mer d'Alboran).

\section{Conclusion}

La région d'Al Hoceima a enregistré des séismes majeurs ayant entrainé des dégâts importants aussi bien sur le plan humain que matériel. Les mécanismes au foyer de ses séismes proposent deux plans nodaux de direction NE-SW à NNE-SSW et NW-SE à WNW-ESE. Les déformations de surface cartographiées lors des séismes de 1994 et 2004 sous forme de fentes de tension organisées en échelon présentent une direction NNESSW à NE-SW [4]. Les données GPS indiquent un mouvement de convergence des plaques Afrique-Eurasie de direction NNW-SSE à NW-SE, avec un échappement du bloc du Rif central vers le SSW le long d'une faille de direction NE-SW à composante décrochante senestre [27]. Pour avoir plus d'information afin de trancher sur la direction de la direction des failles responsable en profondeur du déclanchement des séismes de 1994 et 2004, nous avons analysé les déformations cassantes dans les formations plio_quaternaires de la région d'Al Hoceima. La rosace des failles mesurées indique que la direction NNE-SSW à NE-SW est la plus prédominante. Nous proposons de retenir cette direction comme celle des failles responsables des séismes de 1994 et 2004. Ces failles se prolongent en mer avec une légère déviation pour rejoindre les accidents actifs de la ride d'Alboran responsables du séisme de 2016.

\section{References}

1. D. F. Argus, R. G. Gordon, C. DeMets \& S. Stein. Closure of the Africa $\square$ Eurasia $\square$ North America plate motion circuit and tectonics of the Gloria fault. Journal of Geophysical Research: Solid Earth, 94(B5), 5585-5602. (1989)

2. T. El Mrabet. Les grands séismes dans la région maghrébine (Doctoral dissertation, Thèse d'état, Faculté des lettres et des sciences humaines, Université Mohammed V. Rabat, 435 pp., \{(in arabe)\}). (2005)

3. M. Meghraoui. La faille sismique et ses implications pour l'évaluation des risques sismiques le long de la limite des plaques en Afrique du Nord. En conférence euro-méditerranéenne pour l'intégration de l'environnement(pp. 37-40). Springer, Cham. (2017, novembre).

4. L. Ait Brahim et al. Structural analysis and interpretation of the surface deformations of the February 24th, 2004 Al Hoceima earthquake. Centre Sismologique Euro-Méditerranéen European- 
Mediterranean Seismological Centre www. emsccsem. (2004).

5. A. Poujol, J.F. Ritz, A. Tahayt, P. Vernant, M. Condomines, P. H. Blard, P. H \& A. K. Idrissi. Active tectonics of the Northern Rif (Morocco) from geomorphic and geochronological data. Journal of Geodynamics, 77, 70-88. (2014).

6. A. Calvert et al. An integrated geophysical investigation of recent seismicity in the Al-Hoceima region of north Morocco.. Bulletin of the Seismological Society of America 87, p. 637-651. (1997).

7. C. Ziyadin, M. Meghraoui, M. Akoglu, M., et al. Surface deformation associated with the Mw 6.4, 24 February 2004 Al Hoceima, Morocco, earthquake deduced from InSAR: implications for the active tectonics along North Africa. Bulletin of the Seismological Society of America, vol. 96, no 1, p. 59-68. (2006).

8. O. Azzouz. Lithostratigraphie et tectonique hercynienne des terrains paléozoïques ghomarides du Massif des Bokoya (Rif Interne, Maroc), Thèse 3ème cycle, Univ Mohammed V, Fac. Sci. Rabat. (1992).

9. T. Mourier, Etude géologique et structurale du massif des Bokoya, Trav. Lab. Géol. de l'Afrique, 6. Univ. Paris Sud. (1982).

10. L. Ait Brahim L, B. Tadili, C. Nakhcha, I. Mouayn, M. Ramdani, M. Limouri. Using active faults and seismicity for the strong motion modeling in the Eastern Rif (Northern Morocco). Pure and Applied Geophysics,: Volume 161, Numbers 5-6, pp. 10811091. (2002)

11. A. Chalouan, Ouazani-Touhami, Les failles normales à faible pendage du Rif interne (Maroc) et leur effet sur l'amincissement crustal du domaine d'Alboran. Geogaceta 17, 107-109. (1995).

12. L. Ait Brahim. Tectoniques cassantes et états des contraintes récents au Nord du Maroc. Contribution al'étude du risque sismotectonique. These Doctorat d'Etat, Université Mohammed V, Rabat. (1991).

13. L .Ait Brahim,P. Chotin, M. Ramdani. Failles actives dans le Rif central et oriental (Maroc) - C. R. Acad. Sci. Paris, t. 310, série II, pp. 1123-129. (1990)

14. J. Galindo-Zaldívar, O. Azzouz, A. Chalouan, A. Pedrera, P. Ruano, A. Ruiz-Constán \& M. Benmakhlouf. Extensional tectonics, graben development and fault terminations in the eastern Rif (Bokoya-Ras Afraou area). Tectonophysics, 663, 140-149. (2015).

15. L. Ait Brahim. Cadre sismotectonique et risque sismique de la chaîne rifaine,. Travaux de l'Institut Scientifique, série géographique physique et géologie, $\mathrm{n}^{\circ} 397$ pp.121-135. (2002)

16. B. Deffontaines, P. Chotin, L. A. Brahim \& M. Rozanov. Investigation of active faults in Morocco using morphometric methods and drainage pattern analysis. Geologische Rundschau,81(1), 199-210. (1992)

17. L. Ait Brahim, C. Nakhcha, B. Deffontaines, H. Sami, P. Chotin. Apports de l'analyse conjointe du réseau hydrographique et d'un modèle numérique de terrain pour l'étude de la néotectonique du Rif oriental, (région d'Al Hoceima - Nador, Maroc NE). Revue Télédétection- photo interprétation. Editions ESKA, France $\mathrm{N}^{\circ}$ 4, pp.13-49. (2004)

18. L. Ait brahim, B. Tadili, M. Ramdani, O. Ibn ibrahim. Cadre structural et manifestation de surface du séisme d'Al Hoceima du 26/5/94. poster, 33 rd Meeting of the General committe of the international council of scientific union (ICSU) 11-17 OctobreRabat. (1994)

19. S. O. El Alami, B. Tadili, T. E. Cherkaoui, F. Medina, M. Ramdani, L.A. Brahim \& M. Harnafi. The Al Hoceima earthquake of May 26, 1994 and its aftershocks: a seismotectonic study. (1998)

20. M. Bezzeghoud \& E. Buforn. Source parameters of the 1992 Melilla (Spain, MW=4.8), 1994 Alhoceima (Morocco, MW= 5.8), and 1994 Mascara (Algeria, $\mathrm{MW}=$ 5.7) earthquakes and seismotectonic implications. Bulletin of the Seismological Society of America, 89(2), 359-372. (1999)

21. A. M. Akoglu, Z. Cakir, M. Meghraoui, S. Belabbes, S. O. El Alami, S. Ergintav,\& H. S. Akyüz. The 1994-2004 Al Hoceima (Morocco) earthquake sequence: Conjugate fault ruptures deduced from InSAR. Earth and Planetary Science Letters, 252(3), 467-480. (2006)

22. D. Stich, FDL. Mancilla, D. Baumont et J. Morales. Analyse des sources du tremblement de terre Mw 6.32004 Al Hoceima (Maroc) en utilisant des fonctions de temps source apparentes régionales. Journal of Geophysical Research: Terre solide, 110 (B6). (2005)

23. L. Dorbath, Y. Hahou, B. Delouis, C. Dorbath, J. Van Der Woerd, S. Bardrane, P. Tapponnier. Études sismologiques sur le séisme D'al Hoceima: localisation, et mécanisme du choc principal et des répliques, contraintes et structure de la zone epicentrale. In Colloque Intenational Seisme d'Al Hoceima: bilan et perspectives (pp. 24-26). (2005)

24. J. Van Der Woerd, C. Dorbath, F. Ousadou, L. Dorbath, B, E. Jacques \& H. Haessler. The Al Hoceima Mw 6.4 earthquake of 24 February 2004 and its aftershocks sequence. Journal of Geodynamics, 77, 89-109. (2014).

25. C. Ziyadin, M. Meghraoui, M. Akoglu et al. Surface deformation associated with the Mw 6.4, 24 February 2004 Al Hoceima, Morocco, earthquake deduced from InSAR: implications for the active tectonics along North Africa. Bulletin of the Seismological Society of America, vol. 96, no 1, p. 59-68. (2006).

26. M. Lafosse, E. d'Acremont, A. Rabaute, B. Mercier de Lépinay, A. Tahayt, A. Ammar \& C. Gorini. Evidence of Quaternary transtensional tectonics in the Nekor basin (NE Morocco). Basin Research, 29(4), 470-489. (2017)

27. A. Koulali, D. Ouazar, A. Tahayt, R. W. King, P. Vernant, R. E. Reilinger \& N. Amraoui. New GPS constraints on active deformation along the AfricaIberia plate boundary. Earth and Planetary Science Letters, 308(1), 211-217. (2011). 